\title{
Distributed Deployment Scheme for Homogeneous Distribution of Randomly Deployed Mobile Sensor Nodes in Wireless Sensor Network
}

\author{
Ajay Kumar ${ }^{1}$, Vikrant Sharma ${ }^{2}$ and D. Prasad ${ }^{3}$ \\ ${ }^{1,2,3}$ Department of Computer Science and Engineering, M.M. University, Mullana, \\ Ambala, Haryana, India.
}

\begin{abstract}
One of the most active research areas in wireless sensor networks is the coverage. The efficiency of the sensor network is measured in terms of the coverage area and connectivity. Therefore these factors must be considered during the deployment. In this paper, we have presented a scheme for homogeneous distribution of randomly distributed mobile sensor nodes (MSNs) in the deployment area. The deployment area is square in shape, which is divided into number of concentric regions centered at Base Station, these regions are separated by half of the communication range and further deployment area is divided in to numbers of regular hexagons. To achieve the maximum coverage and better connectivity MSNs will set themselves at the center of the hexagon on the instruction provided by the BS which is located at one of the corner in the deployment area. The simulation results shows that the presented scheme is better than CPVF and FLOOR schemes in terms of number of MSNs required for same coverage area and average movement required by MSNs to fix themselves at the desired location and energy efficiency.
\end{abstract}

Keywords-Active MSNs; Desired location; Candidate location; Communication range; Sensing range etc.

\section{INTRODUCTION}

A wireless sensor network typically consists of a base station (BS) and a group of geographically distributed sensor nodes (SNs) [1]. The SNs are typically small wireless devices with limited computational power, radio transmission range, storage size and battery power that cooperatively perform the task of collecting relevant data and monitor its surrounding for some change or event to occur [2]. The WSNs has its own features that not only differentiate it from other wireless networks but also craft the scope of wireless applications to disaster relief, military surveillance, habitat monitoring, target tracking and in many civic, medical and security applications [3-6]. The SNs may be left unattended in any hostile environment such as battlefields, volcanoes etc., which makes it difficult or sometimes impossible to recharge or replace their batteries. Thus, efforts must be employed to remove this deficiency of WSNs. Many protocols existing in the literature minimize energy consumption on routing paths [3-6]. Even though these approaches increase energy efficiency, they do not always prolong network lifetime; if certain nodes become popular, commonly termed as "hot spots" and present on most of the forwarding paths towards sink in the network. Some of the common characteristics of WSNs that were kept in mind before developing the scheme are discussed in [2]. Some of the major issues in the node deployment strategies are: the area covered by the SNs, connectivity among them, finding the dead SNs in the deployment area, beside these the SNs are fault prone devices due to battery loss or some physical damage during their random distribution from flying BS like helicopter, aero plane etc [7-9]. To avoid such problems, the researchers tried to identify the feasible SNs deployment strategies. However, if ample amount of energy is present to provides mobility to the SNs in the network, the randomly deploy $\mathrm{SNs}$ can be distributed evenly in the deployment area.

One of the most active research areas in wireless sensor networks is the coverage. Coverage is usually interpreted as how well a sensor network will monitor a field of interest. Coverage can be measured in different ways depending on the applications. In addition to coverage it is important for a sensor network to maintain connectivity. Connectivity can be defined as the ability of the sensor nodes to reach the data sink. If there is no available route from a sensor node to the data sink then the data collected by that node cannot be processed. Each node has a communication range which defines the area in which another node can be located in order to receive data. This is separate from the sensing range which defines the area a node can observe. The two ranges may be equal but are often different.

There are several factors that must be considered during the deployment of sensor networks. Many of these will be dependent upon the particular application that is being addressed. The capabilities of the sensor nodes that are being used must also be considered. Most researchers focus on a single deployment model but there are papers that attempt to develop a more general algorithm that can be used in many types of deployment.

Rest of the paper is organized as follows. Section 2 summarizes the literature survey. In Section 3 System Model is presented and Section 4 consists of network model. Network setup is explained in section 5 followed by implementation of scheme in section 6. Finally simulation results and discussion of the work is presented in section 7 followed by conclusion and future work in section 8 . 


\section{LITERATURE SURVEY}

In hostile environment where reach-ability is not possible sensor nodes are deployed randomly from some flying object like helicopter, aero plane etc. In case of random deployment to provide better connectivity Mobile sensors can be used which can relocate themselves into a network.

According to the algorithm in [10], each sensor node determining the location it needs to move to in order to provide maximum coverage. The authors perform several experiments to determine how well the network covers the area and the deployment time of the algorithm. The key weakness in this algorithm is that each node must be within the sensing range of another node in order to determine the optimal location it needs to move to, if a node is not seen by any other nodes then that node cannot determine its relative location.

In [11] authors have presented the virtual force algorithm (VFA) as a practical approach for sensor deployment. The VFA algorithm uses a force-directed approach to improve the coverage provided by an initial random placement. The VFA algorithm offers a number of important advantages. These include negligible computation time and a one-time repositioning of the sensors. Moreover, the desired sensor field coverage and model parameters can be provided as inputs to the VFA algorithm, thereby ensuring flexibility. The VFA algorithm can be made more efficient if it is provided with the theoretical bounds on the number of sensors needed to achieve a given coverage threshold. Also, there is no route plan for repositioning the sensors in the VFA algorithm, where sensor collision can happen during the repositioning. Since the current target localization algorithm considers only one target in the sensor field, it is necessary to extend the presented approach to facilitate the localization of multiple objects. Another extension lies in distributed localization and querying. Extensions to nonmobile sensor nodes and situations of sensor node failures may also be considered.

In [12] authors have presented a new scheme that are not governed by these assumptions, and thus adapt to a wider range of application scenarios. The schemes are designed to maximize sensing coverage and also guarantee connectivity for a network with arbitrary sensor communication/sensing ranges or node densities, at the cost of a small moving distance. The schemes do not need any knowledge of the field layout, which can be irregular and have obstacles/holes of arbitrary shape. Scheme is an enhanced form of the traditional virtual-force-based method, which author term the Connectivity-Preserved Virtual Force (CPVF) scheme. Authors show that the localized communication, which is the reason for its simplicity, results in poor coverage in certain cases. To improve the performance further authors described a Floor based scheme which overcomes the difficulties of CPVF and, as a result, significantly outperforms it and other state-of-the-art approaches.
In [13] authors have presented a potential field based mobile sensor network deployment strategy. Two key ideas that were dealt with in [13] are (i) forming a hexagonal structure with artificial forces generated from a potential field and (ii) its hierarchical application for wider area coverage. Hexagonal formation is shown to the optimal placement for identical sensor model in terms of coverage area. Potential field based artificial force algorithm provides a simple and efficient method to deploy large number of sensors because the force is used as control input for each node without any sophisticated control algorithms. This aspect enables constructing a hierarchical structure without any additional complexity. The main weakness of this scheme is that it cannot achieve global optimization. This is a fundamental characteristic of the potential field based method. In some cases, they have undesirable formations, where a coverage holes exist in the middle of the hexagonal structure.

While focusing on the problems of coverage, existing deployment schemes largely oversimplify the conditions for network connectivity. These schemes either assume that the communication range is large enough for sensors in geometric neighborhoods to obtain location information through local communication, or assume a dense network that remains connected. In this research work we will propose a deployment scheme for mobile wireless sensor networks to support optimum coverage, while maintaining the connectivity.

\section{SySTEM MODEL}

Base Station is considered to have unlimited energy with powerful transmitters (i.e. unidirectional or Omni directional) depending on the location of Base Station, which can transmit query packets within deployment area by broadcasting or multicasting. As Shown in Fig. 1; Base Station directly sends Request to specific node using its long communication range, sensor node in turn replies to the Base Station by sending a response packet using multihop communication (node to node communication).

The proposed model focuses on deployment of sensors nodes to achieve following results:

1) Maximum coverage with minimum number of sensors: The desired location for the placement of MSNs is computed by Base Station such that there is minimum overlapping of sensing range $\left(\mathrm{r}_{\mathrm{s}}\right)$ of adjacent MSNs.

2) Minimizing the average movement performed by MSNs: The randomly deployed MSNs are assigned their final positions in deployment area by Base Station in such a manner that minimum amount of movement is required. Minimizing the movement further minimizes power consumption.

3) Minimizing the inter node communication during deployment : The movement and placement of MSNs to particular location is decided and guided by Base Station, so inter node communication required for determining desired location for particular MSN is exempted.

of sensors hence making the whole system energy efficient, reliable, cost effective, and long lasting. 


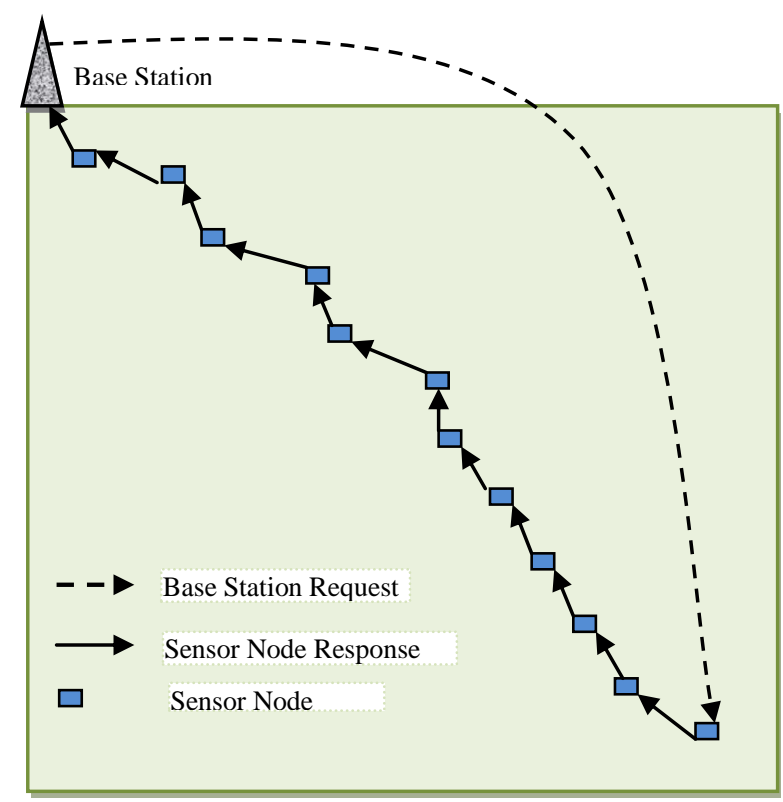

Fig. 1. Base Station Request-Response model.

4) Minimizing the energy required during deployment: All the above approaches aims at minimizing the power consumption Minimizing the energy required during deployment: All the above approaches aims at minimizing the power consumption

\section{NETWORK MODEL}

In the proposed scheme all the sensor nodes are mobile in nature. The sensor nodes are having same communication range, sensing range and computation power. The deployment area is square in shape, which is divided into number of regions these regions are separated by half of the communication range further deployment area is divided into numbers of regular hexagons. MSNs will set themselves at the center of the hexagon on the instruction provided by the BS and BS is located at one of the corner in the deployment area. We have made assumptions that all the sensor nodes fall within the deployment area when deployed randomly and the total number of SNs deployed is greater than or equal to the total number of desired locations in the deployment area. Following are the various issues in the random deployment:

\section{A. Inefficient utilization of resources}

Random deployment of sensor nodes lead to overlapping of sensing range of multiple sensors, where as some patches in deployment area remain uncovered (these patches are not in sensing range of any sensor nodes).

\section{B. Redundant data generation}

Overlapping of sensing range of multiple sensors lead to generation of packets with redundant data, which leads to congestion in the network, hence utilizes more power resources (battery).
Random deployment requires large number of sensor nodes to achieve required level of coverage which increases the system cost. So for optimum and efficient utilization of resources and for better control and management, homogenous deployment is preferred.

\section{NETWORK SETUP}

The sensor nodes are randomly deployed in the deployment area to be monitored by some flying object like aero plane, helicopter etc. as shown in Fig. 2.

The network setup of the presented model is divided in to the followings two phases:

\section{A. Pre deployment Phase}

Before the deployment of MSNs in the deployment area the following operations are performed at the BS.

Computation of Communication range $r_{c}$ and Sensing range $r_{s}$ : Let $\mathrm{s}$ is length of side of regular hexagon. To avoid uncovered region in the deployment area the, the sensing range $\left(r_{s}\right)$ of the MSNs should be equal to $s$ at least. The

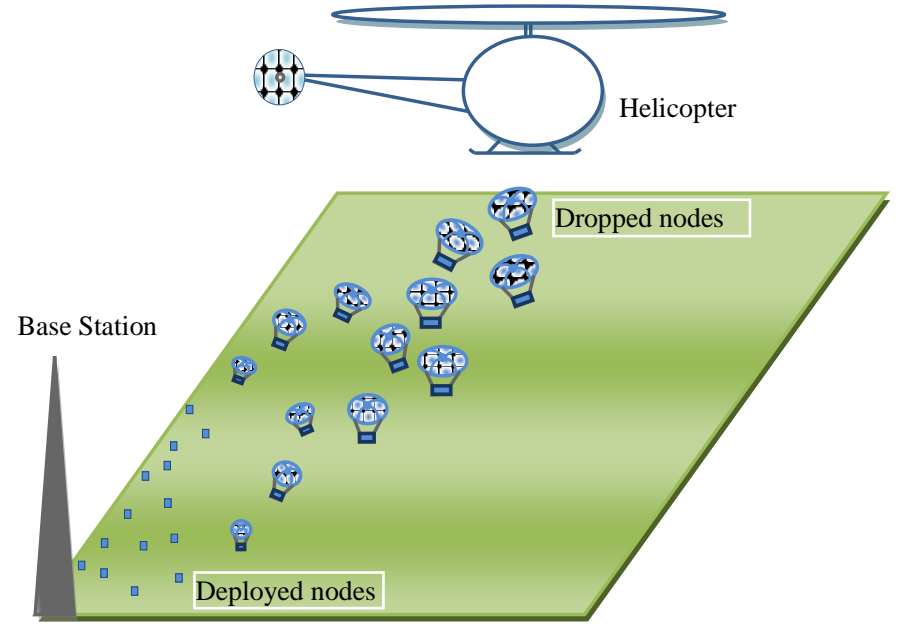

Fig. 2. Random deployment of sensor nodes using flying machine.

communication range $\left(r_{c}\right)$ of the MSNs should be equal to the distance between the centers of the adjacent regular hexagon at least. The relationship between sensing range $\left(r_{s}\right)$ and communication range $\left(r_{c}\right)$ can be derived as follows:

Let $\mathrm{C}$ and $\mathrm{D}$ are centers of any two adjacent hexagons, $\mathrm{AB}$ is the common side of the above adjacent hexagon and $M$ is the mid point of side $A B$ as shown in the Fig. 3 By the property of regular hexagon, triangle $\mathrm{CAB}$ and $\mathrm{DAB}$ will be equilateral triangle and triangle $\mathrm{AMC}$ will be right angle triangle with $\mathrm{AC}$ as hypotenuse. By the property of right angle triangle we have the following relation: 


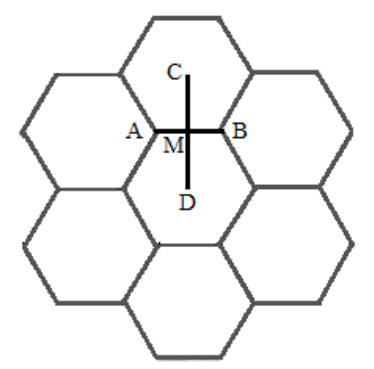

Fig. 3. Coverage pattern of the deployment area.

$$
\mathrm{CM}=\sqrt{\mathrm{AC}^{2}-\mathrm{AM}^{2}}=\sqrt{\mathrm{s}^{2}-(\mathrm{s} / 2)^{2}}=\sqrt{3 \mathrm{~s}^{2} / 4}
$$

Sensing range $r_{\mathrm{s}}$ in our model is equal to the length of side $\mathrm{s}$ and Communication $\mathrm{r}_{\mathrm{c}}$ range will be twice of the $\mathrm{CM}$ i.e. $\mathrm{r}_{\mathrm{c}}=2 * \mathrm{CM} ; \quad \mathrm{r}_{\mathrm{c}}=2 * \mathrm{CM}=\mathrm{s} * \sqrt{3}=\mathrm{r}_{\mathrm{s}} * \sqrt{3}$

1) Logical division of the deployment area: The BS divides the deployment area into $\mathrm{n}$ concentric regions centered at $B S$ referred as $R_{1}, R_{2}, R_{3}, \ldots \ldots, R_{n}$ as shown in the Fig. 4 each separated by half of the communication range $r_{c}$, further entire deployment area is divided in to number of regular hexagons and center points of these hexagons are called desired locations for deployment (these are the locations where SNs will fix themselves).

2) Computation of desired locations in various regions: BS constructs $n$ Lists $\mathrm{RL}_{1}, \mathrm{RL}_{2}, \mathrm{RL}_{3}, \ldots \ldots \ldots, \mathrm{RL}_{\mathrm{n}}$ one for each region containing list of desired location in that region and add these lists to the Queue (QR) as shown in Fig. 5.

\section{B. Post deployment Phase}

The various operation performed in this phase is divided in to the following steps. In this phase MSNs are deployed in the deployment area. After the deployment MSNs find their location as in [14] and maintains at their own end. BS maintains a list called Active_Node_List[] containing Id and current location of MSNs under consideration, which are connected to BS either directly or indirectly.

The BS broadcast an ADV packet containing it own id and location in the deployment area. On receiving the ADV packet, all MSNs computes their own distance $\left(\mathrm{d}_{\mathrm{BS}}\right)$ from the BS, based on $\mathrm{d}_{\mathrm{BS}}$ MSNs determines the region to which they belong, as MSN belongs to region $R_{\mathrm{i}}$ if its $\mathrm{d}_{\mathrm{BS}}$ lies between $(i-1) * 1 / 2 * r_{c}$ and $i * 1 / 2 * r_{c}$ those MSNs for which $d_{B S}$ is less than or equal to $r_{c}$ sends a RPLY packet containing their own id and location to the BS using CDMA to avoid any collision.

The maximum time taken by furthest node in $i^{\text {th }}$ region to come in to the communication range of the placed node (i.e. node in region Front-1) is T.

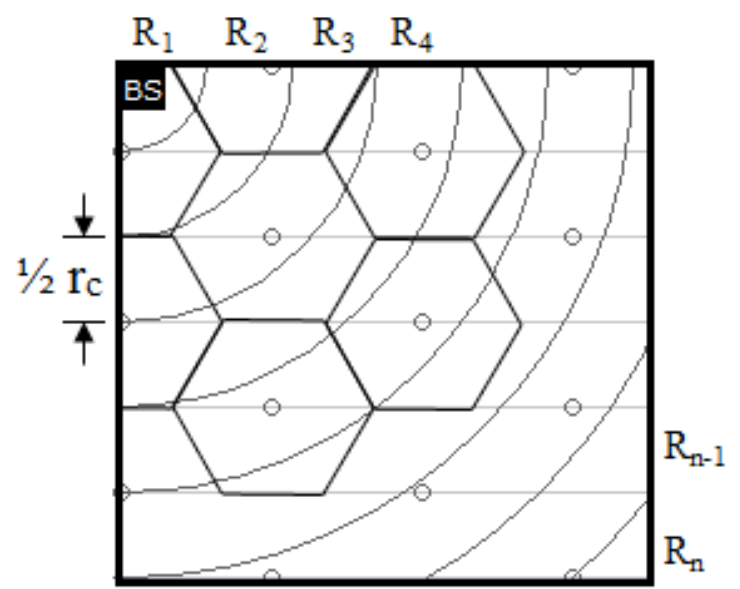

Fig. 4. Logical division of deployment area.

Algorithm 1: Homogenious distribution of MSNs

Step1: On receiving RPLY packet, BS updates Active_Node_List[] with entries of newly connected MSNs.

Step2: BS get all the desired locations from QR[front], Update Selected_Location_List[] = QR[front] and set $\mathrm{i}=$ front;

if (Size of Active_Node_List[] = 0 ) perform Step3 else perform Step 4.

Step 3: $i=i+1$;

BS instruct the various MSNs in the next region (i.e. $\mathrm{R}_{\mathrm{i}}$ ) to move towards the appropriate MSNs in the previous region (i.e. $\mathrm{R}_{\text {front- }-1}$ ) or $\mathrm{BS}$ to get connected by constructing a packet containing Id_Location_ List[] having Id and Locations of all MSNs placed in previous region (ie. $\mathrm{R}_{\text {front- }}$ ${ }_{1}$; if previous region exists) followed by its own Id and Location and send this packet for the MSNs in region $R_{i}$ by specifying the region in packet.

The MSNs compare the region specified in the packet with its own region and matching MSNs compute their distance from all the locations specified in Id_Location List[] contained in the packet and start moving towards the MSNs with $d_{\text {min }}$ or towards BS if $d_{B S}<d_{\min }$ to make themselves connected to the BS somehow and stop moving when they are within the range of placed MSNs or BS towards which they are moving, sends RPLY packet containing their Id and location to the BS. BS waits for time interval T and Perform Step1.

Step 4:

i) BS select the appropriate MSNs (as computed by algorithm 2) from Active_Node_List[] to fill the various locations obtained in Step2, remove the corresponding entry from Active_Node_List[] and remove the allocated locations from $\mathrm{QR}$ [front]

if (Size of $\mathrm{QR}[$ front] = 0) remove $\mathrm{QR}$ [front] from $\mathrm{QR}$ (ie. front $=$ front +1 ); if(QR is empty) then exit. 
ii)BS constructs a packet containing Id_Location_List[] having Id of selected MSNs and location computed for them, followed by Id and location of MSNs placed in previous region (i.e. $R_{\text {front-1 }}$,if any) followed by its own id and location and instruct those MSNs to move to allocated location by sending the above packet to the deployment area. The MSNs in the deployment area compare their own id with the id in the list and whose id matches start moving towards the specified location, set themselves to the specified location and mark themselves as "placed" and the MSNs in the next region (ie. $\mathrm{R}_{\text {front }+1}$ ) computes its distance from all the locations in the packet which are allocated to some MSNs, find the minimum distance $\left(\mathrm{d}_{\min }\right)$ among them and start moving either_towards the MSNs with $d_{\min }$ or towards the BS if $d_{B S}$ is less than $d_{\text {min }}$ to make themselves connected to the BS somehow and stop moving when they are within the range of any placed MSNs or when BS comes within the range of moving MSNs and send RPLY packet containing their Id and location to the BS and then perform Step1.

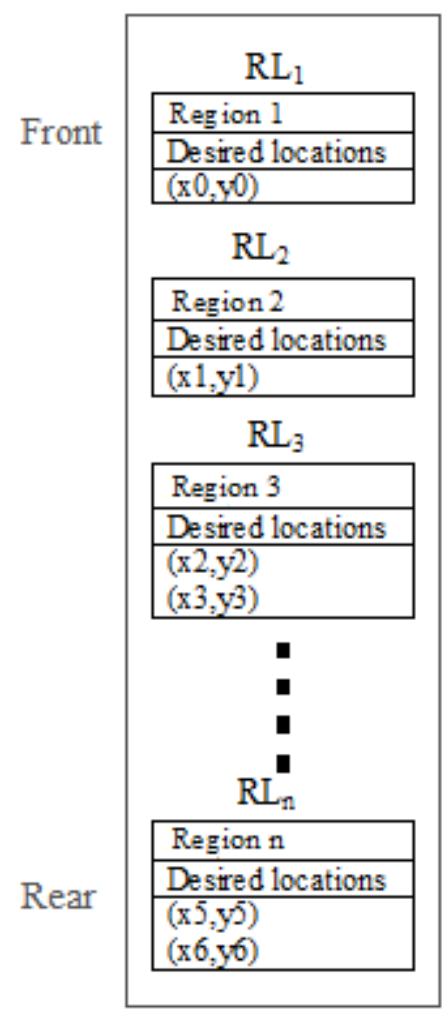

Fig. 5. Queue containing desired locations of all regions.

After all the desired locations are occupied by MSN, the BS instructs all the remaining MSNs to go to sleep mode by sending the SLEEP packet containing Id of all the remaining MSNs. These sleep MSNs may be used to occupy the uncovered spot ( if any) or may be used to replace the dead MSNs in the deployment area on the instruction provided by the BS.

In Fig. 6 green arrow represents the transfer of REPLY packet containing Id and current location of newly connected MSN to Base Station using multi-hop communication, blue arrow represents transmission of packet containing Id_Location_list to selected MSNs from Active_Node_List[] (i.e. unplaced, connected MSNs from region $R_{0}$ to $R_{i}$ ) and to MSNs in region $R_{i+1}$ by Base Station.

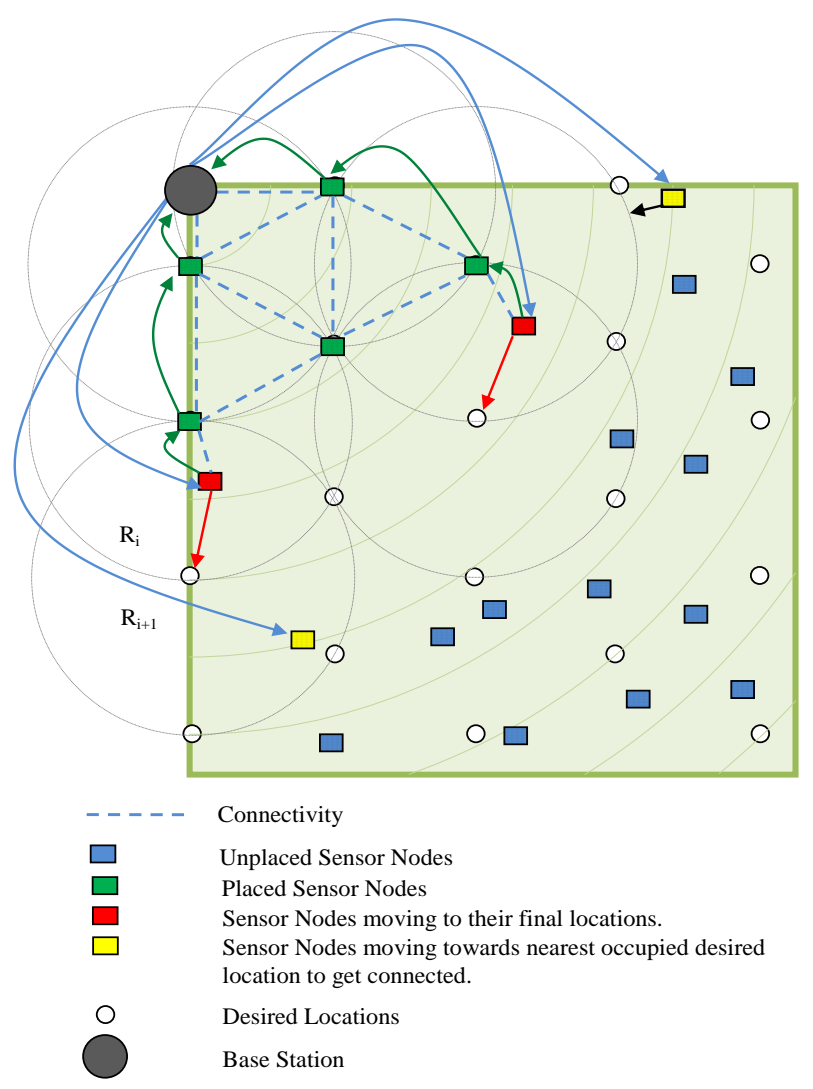

Fig. 6. Placement pattern and sequence of MSNs.

Red arrow represents the movement of selected MSNs to fix themselves to the final location specified to them in Id_Location_List[], black arrow represents the movement of MSNs in region $R_{i+1}$ towards the nearest location in Id_Location_List[] until that location comes within communication range $\left(r_{c}\right)$ of these MSNs, so that connection can be established thorugh the MSN placed at that location.

After the establishment of connection newly connected MSNs sends REPLY packet to Base Station as specified by green arrow.

Algorithm 2: Computing of the Appropriate Location for particular MSN

Step1:BS computes the distance of MSNs in Active_Node_List[] from all the locations in Selected_Location_List[] and construct tables containing candidate Location and their Distance sorted according to distance and store these tables in LD_List[] as shown in Fig. 7 using this list, BS 
constructs Id_Location_List[] as shown in Fig. 8 containing MSNs Id and their Final Location.

Step2: For i $=1$ to Size_of Active_Node_List[] ; repeat Step3 to Step5

Step3:Table ${ }_{\min }=$ first table in the list of location-distance tables

Step4: Take first record from Table min $_{\text {in }}$ and compare its location value with the location value of first record of all the other tables.

Step5: If location value matches

\{

Table $_{\min }=$ table with minimum distance value in first record

Table $_{\text {temp }}=$ other table

If (size of Table temp $_{\text {en }}>1$ )

Remove the first record from Table temp $_{\text {}}$ Else

Remove Table $_{\text {temp }}$ and Go to step 3

\}

Else

\{

Node Id = Id of node whose table is Table $_{\min }$;

Final Location= location value at first record in Table $_{\text {min }}$;

Add Node Id and Final Location to Node-Location Table;

Remove Table $_{\min }$ from list of location-distance tables; \}

\begin{tabular}{|c|c|}
\hline \multicolumn{2}{|c|}{ LD_List] } \\
\hline \multicolumn{2}{|c|}{ LD_List[1] } \\
\hline \multicolumn{2}{|c|}{ Node Id:4 } \\
\hline $\begin{array}{l}\text { Candidate } \\
\text { Location }\end{array}$ & Distance \\
\hline$(460,550)$ & 93.15041 \\
\hline$(580,411)$ & 100.84642 \\
\hline$(400,584)$ & 162.11415 \\
\hline \multicolumn{2}{|c|}{ LD_List[2] } \\
\hline \multicolumn{2}{|c|}{ NodeId:13 } \\
\hline $\begin{array}{l}\text { Candidate } \\
\text { Location }\end{array}$ & Distance \\
\hline$(580,411)$ & 62.072536 \\
\hline$(460,550)$ & 145.11375 \\
\hline$(400,584)$ & 214.03271 \\
\hline \multicolumn{2}{|c|}{ LD_List[3] } \\
\hline \multicolumn{2}{|c|}{ Node Id:58 } \\
\hline $\begin{array}{l}\text { Candidate } \\
\text { Location }\end{array}$ & Distance \\
\hline$(460,550)$ & 45.276924 \\
\hline$(400,584)$ & 112.00893 \\
\hline$(580,411)$ & 153.56107 \\
\hline
\end{tabular}

Fig. 7. List of tables containing Candidate locations and their distance from MSNs under consideration.

\begin{tabular}{|l|l|}
\hline $\begin{array}{l}\text { Node } \\
\text { Id }\end{array}$ & Final Location \\
\hline 58 & $(460,550)$ \\
\hline 13 & $(580,411)$ \\
\hline 4 & $(400,584)$ \\
\hline
\end{tabular}

Fig. 8. List representing Id_Location_List[]

\section{IMPLEMENTATION}

There are a number of robots that can be used as mobile SNs in this scheme, depending on the application and area of usages; like mountains, deserts, plane, etc. For simulation the presented scheme considered the Soldier UGV (SUGV). SUGV is a man- packable small robot system, weighing less than $30 \mathrm{lbs}$, used for Urban Operations environments and subterranean features to remotely investigate the obstacles, structures and the structural integrity of facilities and utilities. The SUGV system is highly mobile for dismounted forces and capable of being re-configured for other missions by adding or removing SNs, modules, mission payloads and subsystems.

\section{SimUlation ResUlts AND Discussion}

The presented model is simulated in JAVA. The coverage area, number of MSNs required and average movement performed by the MSNs to fix themselves to the desired location, are the various parameters we have considered for the comparison of the developed scheme with some existing schemes. The coverage pattern of the proposed scheme and the FLOOR based scheme are presented in Fig. 9 and Fig. 10 respectively. From the result obtained we can observes that the developed scheme requires $280 \mathrm{MSNs}$ to achieve $100 \%$ coverage if the value of the communication range $\left(r_{c}\right)$ and sensing range $\left(r_{s}\right)$ are $70 m$ and $40 m$ respectively, as shown in Fig. 11 , whereas the number of MSNs required to achieve

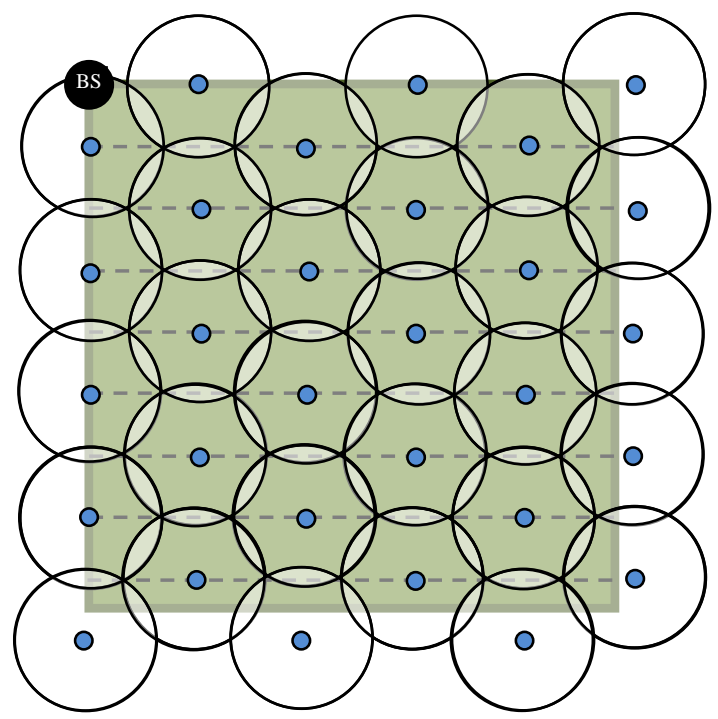

Fig. 9. $100 \%$ coverage by proposed scheme. 
$100 \%$ coverage is increased to 360 when the value of the communication range $\left(r_{c}\right)$ and sensing range $\left(r_{s}\right)$ are $60 \mathrm{~m}$ and $35 \mathrm{~m}$ respectively. The maximum coverage that can be achieved in FLOOR scheme is around 96\% if the value of the communication range $\left(r_{c}\right)$ and sensing range $\left(r_{s}\right)$ are $60 \mathrm{~m}$ and $60 \mathrm{~m}$ respectively and requires $300 \mathrm{MSNs}$, this is due to uncovered patches in deployment area as shown in Fig. 10.

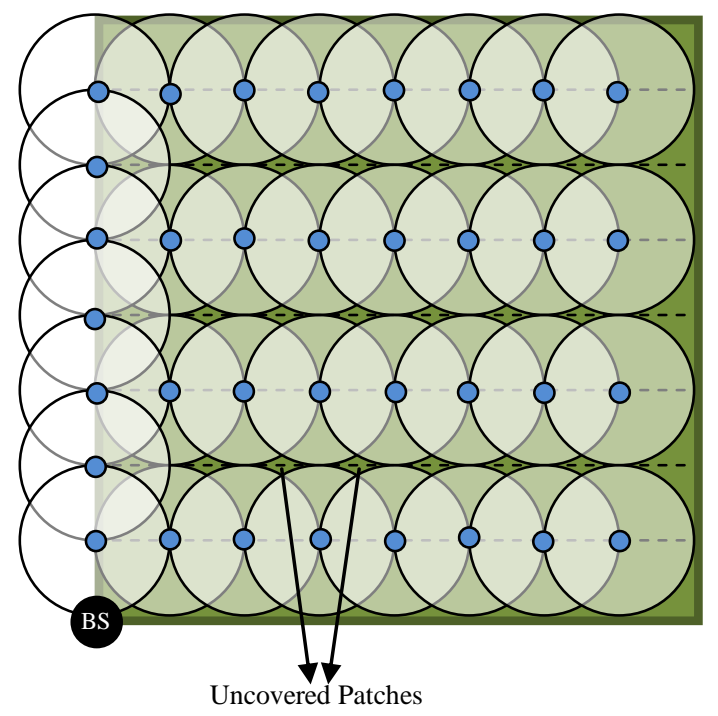

Fig. 10. Uncovered patches left in FLOOR based scheme.

Whereas the maximum coverage that can be achieved in CPVF is around $93 \%$ for the same value of the communication range $\left(r_{c}\right)$, sensing range $\left(r_{s}\right)$ and the number of MSNs required as in FLOOR scheme.

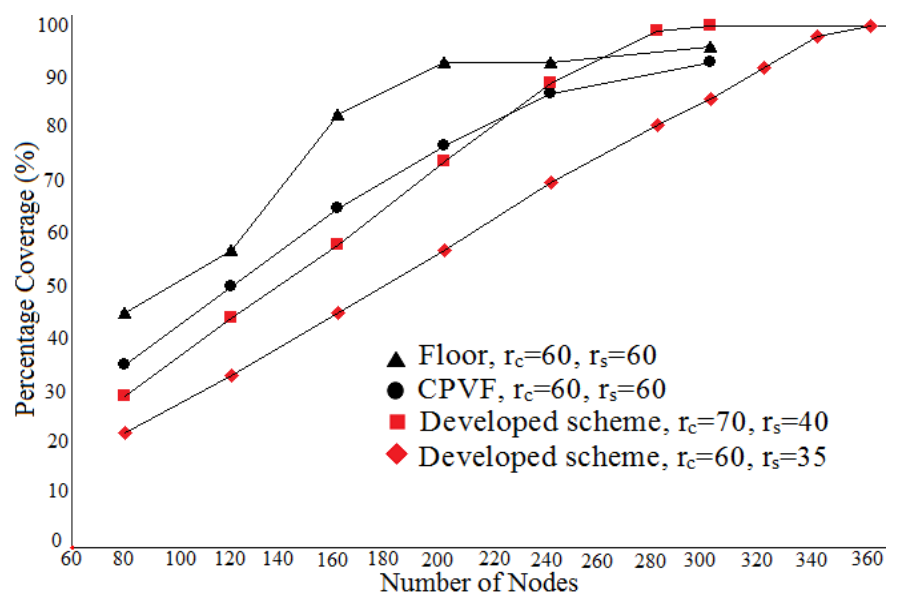

Fig. 11. Comparision of Percentage coverage versus number of Nodes

The average movement takes place by MSNs to set themselves at the appropriate location is shown in Fig. 12. We can observe that for 80 numbers of MSNs the average movement performed by the MSNs in the developed scheme is around $200 \mathrm{~m}$ which is approximately equal to the FLOOR scheme and approximately half of the CPVF scheme. We can also observe that as the number of MSNs are increases, the average movement performed by MSNs decreases in the developed scheme whereas in FLOOR scheme and CPVF scheme as the number of MSNs increases the average movement also increases and at a value of 200 number of MSNs it becomes 330m in Floor scheme, $1060 \mathrm{~m}$ in CPVF scheme and only $148 \mathrm{~m}$ in the developed scheme when communication range $\left(r_{c}\right)$ and sensing range $\left(r_{s}\right)$ are $70 \mathrm{~m}$ and 40 respectively and the average movement $158 \mathrm{~m}$ for the value of communication range $\left(r_{c}\right)$ and sensing range $\left(r_{s}\right)$ equal to $60 \mathrm{~m}$ and $35 \mathrm{~m}$ respectively.

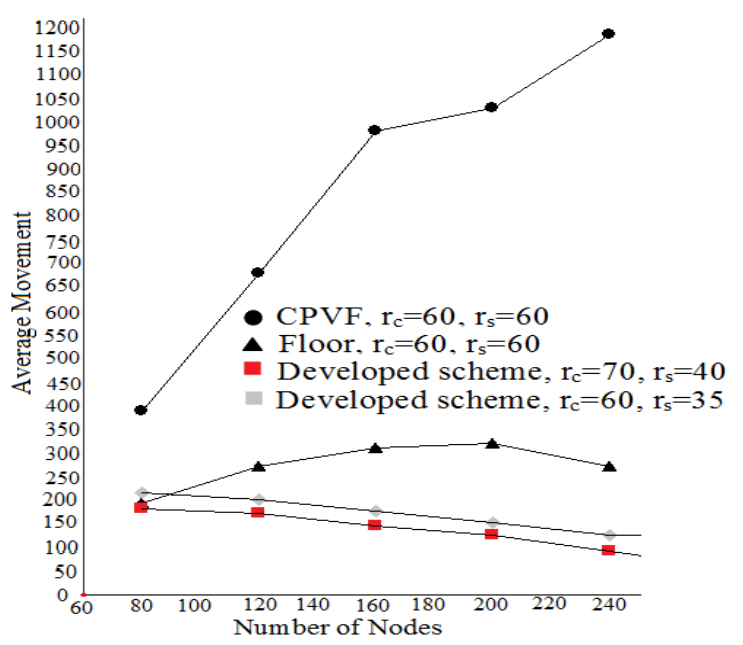

Fig. 12. Comparison of average movement versus number of nodes.

\section{CONCLUSION AND FUTURE WORK}

In this work we have proposed a scheme for homogeneous distribution of randomly deployed MSNs to achieve maximum coverage while maintaining connectivity. The proposed scheme is energy efficient as movement of MSNs during deployment is minimized (energy consumed is directly proportional to the movement) and inter MSNs communication required for their homogenous distribution, is minimized to great extent by using Base Station to guide MSNs to set themselves to their final locations. The simulation result shows that the performance of the developed scheme is better than the earlier work.

In this scheme we have considered square deployment area and obstacle is ignored. As a future work one may think a deployment area of any irregular shape and introduce obstacle in the deployment area.

\section{REFERENCES}

[1]. D. P. Agrawal and Q-A. Zeng, "Introduction to Wireless and Mobile Systems”, Brooks/Cole publisher,2003.

[2]. I. F. Akyildiz, W. Su, Y. Sankarasubramaniam, and E. Cayirci, "Wireless sensor networks: A survey", Computer Networks Journal, Elsevier Science, Vol. 38(4):393-422, No. 4 pp 393-422, March 2002.

[3]. Matt Welsh, Dan Myung, Mark Gaynor, and Steve Moulton. "Resuscitation monitoring with a wireless sensor network". In Supplement to Circulation: Journal of the American Heart Association, October 2003. 
[4]. G.L. Duckworth, D.C. Gilbert, and J.E. Barger. "Acoustic counter-sniper system", In SPIE International ymposium on Enabling Technologies for Law Enforcement and Security, 1996.

[5]. Alan Mainwaring, Joseph Polastre, Robert Szewczyk, and David Culler. "Wireless sensor networks for habitat monitoring", In First ACM International Workshop on Wireless Sensor Networks and Applications, 2002.

[6]. Robert Szewczyk, Joseph Polastre, Alan Mainwaring, and David Culler. "Lessons from a sensor network expedition", In First European Workshop on Wireless Sensor Networks (EWSN'04), January 2004.

[7]. H. Zhang and J. C. Hou. Maintaining sensing coverage and connectivity in large sensor networks. Intl. Journal of Wireless Ad Hoc and Sensor Networks, 1(1-2):89-124, 2005.

[8]. F. Ye, G. Zhong, S. Lu, and L. Zhang, "Peas: A robust energy conserving protocol for long-lived sensornetworks," in Proceedings of the 10th IEEE International Conference on Network Protocols, Washington, DC, USA, 2002. pp. 200-201.

[9]. Siqueira, M. Fiqueiredo, A. Loureiro, J. Nogueira, and L.Ruiz, "An integrated approach for density control and routing in wireless sensor networks", in Proceedings of Parallel and Distributed Processing Symposium, Greece, April 2006, pp. 10-19.
[10]. Howard, M. J. Matari'c, and G. S. Sukhatme, "An incremental self deployment algorithm for mobile sensor networks," Autonomous Robots, vol. 13, no. 2, pp. 113-126, Sep. 2002.

[11]. Yi Zou and Krishnendu Chakrabarty, "Sensor Deployment and Target Localization Based on Virtual Forces", IEEE INFOCOM 2003.

[12]. Guang Tan, Member, IEEE, Stephen A. Jarvis, Member, IEEE, and Anne-Marie Kermarrec, "Connectivity-Guaranteed and ObstacleAdaptive Deployment Schemes for Mobile Sensor Networks", IEEE TRANSACTIONS ON MOBILE COMPUTING, VOL. 8, NO. 6, JUNE 2009.

[13]. Jaeyong Lee, Avinash D. Dharne and Suhada Jayasuriya, "Potential Field Based Hierarchical Structure for Mobile Sensor Network Deployment", Proceedings of the 2007 American Control Conference Marriott Marquis Hotel at Times Square New York City, USA, July 1113, 2007.

[14]. Baoli Zhang and Fengqi Yu "An Energy Efficient Localization Algorithm for Wireless Sensor Networks Using a Mobile Anchor Node", In Proceedings of the 2008 IEEE International Conference on Information and Automation June 20 -23, 2008, Zhangjiajie, China. 